\title{
ESTUDIO SOBRE LA PELIGROSIDAD DEL FENÓMENO DE VIENTOS FUERTES EN ESPAÑA
}

\author{
(STUDY OF THE STRONG WINDS PHENOMENON HAZARD IN SPAIN)
}

M. ${ }^{\text {a }}$ Teresa Piserra, Lic. en Ciencias Geológicas

Fecha de recepción: $22-\mathrm{X}-92$ Javier del Río, Actuario

$113-27$

MAPFRE

\section{RESUMEN}

En general, los estudios sobre el viento en España se analizan desde la óptica de la viabilidad de su aprovechamiento energético. Para ello, es preciso observar y cuantificar la velocidad, dirección y frecuencia de los vientos predominantes en cada zona, ya que éstos son los que, por su constancia, hacen posible su explotación energética.

Sin embargo, el estudio desarrollado a continuación se enfoca en un sentido diferente, ya que pretende analizar la capacidad destructiva del viento, mediante el tratamiento estadistico de las series de rachas máximas registradas en las diferentes estaciones climatológicas.

El resultado final es un mapa de peligrosidad de viento por provincias, que constituye una primera aproximación al conocimiento de la intensidad del fenómeno del viento, desde la perspectiva de sus manifestaciones más extremas.

\section{SUMMARY}

Generally speaking, the studies on the wind in Spain are carried out from the point of view of the feasibility of the exploitation of its energy. In order to archieve this aim it is necessary to observe and quantify the speed, direction and frequence of the winds predominant in each area, since these are the winds which, due to their constancy, allow the exploitation of their energy.

Nevertheless, the following study takes a different approach. It tries to analyze the destruction capacity of the wind by means of a statistical treatment of a series of maximum gusts registered in the different climatological stations.

The final result is the map of wind hazard by provinces, which represents an initial approximation to the knowledge of wind phenomenon intensity studied through its strongest manifestation.

\section{Introducción}

En principio, se puede afirmar que España es una zona poca ventosa, ya que no queda englobada en las áreas de influencia de los ciclones tropicales y, a nivel general, no se ve afectada por los fuertes gradientes de presión derivados de la situación atmosférica normal a lo largo del año. Sin embargo, existen registros históricos de velocidades respetables, como los $187 \mathrm{~km} / \mathrm{h}$ medidos en el observatorio del Monte Igueldo en San Sebastián (Guipúzcoa) el 15 de enero de 1975 y los $196 \mathrm{~km} / \mathrm{h}$ registrados en el observatorio de Izaña (Tenerife) el 25 de febrero de
1947 como máxima velocidad medida en el territorio español. La máxima velocidad peninsular deducida ha sido de $190 \mathrm{~km} / \mathrm{h}$ en el observatorio de Oviedo, donde el instrumental fue arrastrado por el viento.

Como caso particular, existen referencias de un evento ocurrido el 15 de febrero de 1941. Un violento temporal arrasó el oeste peninsular, aunque también quedó reflejado en algunos observatorios del sur y este de la Península. La escaseź de aparatos registradores repartidos por toda Espãna no permitió calibrar los efectos del temporal en todo el territorio nacional. 
A continuación, se presentan algunas velocidades que pudieron registrarse entonces, comparadas con otros datos de velocidades recogidos posteriormente en las mismas estaciones, observándose que aquellos máximos históricos pueden superarse.

\begin{tabular}{|l|c|c|c|}
\hline OBSERVATORIOS & $\begin{array}{c}\text { VELOCIDAD RACHA } \\
\text { MÁXIMA } \\
15-2-1941\end{array}$ & \multicolumn{2}{|c|}{$\begin{array}{c}\text { RACHAS MÁXIMAS } \\
\text { POSTERIORES }\end{array}$} \\
\cline { 3 - 4 } & $126 \mathrm{~km} / \mathrm{h}$ & $124 \mathrm{~km} / \mathrm{h}$ & $\begin{array}{r}25-12-58 \\
1-12-59\end{array}$ \\
\hline ALMERÍA & $119 \mathrm{~km} / \mathrm{h}$ & $130 \mathrm{~km} / \mathrm{h}$ & $25-2-59$ \\
\hline LEÓN & $112 \mathrm{~km} / \mathrm{h}$ & $117 \mathrm{~km} / \mathrm{h}$ & $25-2-89$ \\
\hline LOGROÑO & $180 \mathrm{~km} / \mathrm{h}$ & $187 \mathrm{~km} / \mathrm{h}$ & $15-1-89$ \\
\hline SAN SEBASTIÁN & (No registracla) & $148 \mathrm{~km} / \mathrm{h}$ & $28-12-51$ \\
\hline SANTANDER & & & \multicolumn{3}{|l}{} \\
\hline
\end{tabular}

Es muy probable que el dato de velocidad en Santander el 15 de febrero de 1941 fuera superior al de San Sebastián, pero fue imposible registrarlo porque los aparatos quedaron destrozados.

En general, los vientos fuertes en España acompañan temporales o tormentas locales, son racheados y poco constantes. En los observatorios del interior, las máximas velocidades se registran en verano. En los observatorios cercanos a la costa cantábrica, los máximos se presentan en invierno y en los observatorios de la costa mediterránea, los máximos son en primavera y otoño.

\section{Antecedentes}

Para abordar el estudio de los vientos fuertes en España, se ha consultado la información cartográfica siguiente:

- Mapa de zonas eólicas de NTE-ECV, 1988

Establece cuatro niveles de peligrosidad (w, x, y, z) de menor a mayor y delimita sobre la Península dos núcleos de máxima peligrosidad: uno centrado sobre las provincias de Salamanca y Zamora y otro sobre la Cordillera Pirenaica.

- Mapa Eólico Nacional (Instituto de Meteorología INM, 1988)

Constituye un análisis del viento en España para aplicación a la prospección eólica realizado con datos de 57 observatorios de la red de estaciones del INM.
- Mapa del Recorrido Medio del Viento (Font, 1983)

Refleja las isotacas o líneas de igual valor de velocidad del viento, correspondientes a los valores medios anuales del recorrido del viento en kilómetros por hora. Arbitrariamente, se denominan zonas ventosas aquéllas con velocidad media de viento superior a los $20 \mathrm{~m} / \mathrm{sg}$, por lo que quedan limitadas tres bandas litorales extremas al noroeste, noreste y sur de la Península Ibérica. Se incluyen también unas isletas sobre el valle del Ebro, Sistema Central y La Mancha.

\section{Elaboración del mapa de peligrosidad de vientos fuertes en España}

\subsection{Red Nacional de Estaciones Climatológicas}

El Instituto Nacional de Meteorología (INM) es el organismo público encargado del control y mantenimiento de la red de estaciones distribuidas por todo el territorio nacional. En cada una de ellas se recogen los datos de multitud de variables climatológicas. En particular, la información disponible sobre vientos hace referencia a los siguientes conceptos:

- Dirección dominante del viento.

- Recorrido del viento en km.

- Recorrido medio por día en km.

- Recorrido máximo en un día y fecha.

- Racha máxima del viento en $\mathrm{km} / \mathrm{h}$ y fecha.

De toda esta información, la correspondiente a rachas máximas del viento en $\mathrm{km} / \mathrm{h}$ es la que se ha elegido como factor a considerar a la hora de cuantificar la peligrosidad del viento.

En el Calendario Meteoro-Fenológico de 1951, publicado por el entonces Servicio Meteorológico Nacional, ya se reflejó una tabla de rachas máximas absolutas y una descripción de la red de estaciones dotadas de aparatos medidores (anemocinemógrafos). Este calendario también hace referencia a un estudio similar realizado en 1948 a raíz de las frecuentes consultas de personas procedentes de todos los campos (actuarios, arquitectos, aviadores, ingenieros, marinos y telegrafistas). Todos ellos necesitan este tipo de datos para sus ā̄licaciones, ya sean éstas la elaboración de tarifas, el diseño en la construcción, comunicaciones, transportes, etcétera. 
La distribución de la red de observatorios que aportan datos de rachas máximas de viento, no es aún tan densa y completa como sería necesario, dada la variabilidad del fenómeno del viento en sí. Este inconveniente es asumible teniendo en cuenta que el viento está muy condicionado por la morfología del relieve y la velocidad de las rachas puede variar entre lugares sitos a cortas distancias. La mera presencia de montañas, valles encajonados, bordes de mesetas y otros accidentes orográficos modifica sensiblemente la velocidad del flujo de los vientos.

Para obtener las velocidades de las rachas máximas se utilizan los anemocinemógrafos. Son aparatos de registro continuo de la velocidad del viento que están compuestos por un anemómetro neumático inscriptor, una veleta registradora y un anemómetro registrador de recorrido. El registro de la velocidad de la racha máxima se realiza sobre una banda de papel que recubre un tambor que gira marcando el - tiempo. La veleta está a 10 metros de altura sobre el suelo, aunque el registro se refleja en el interior del observatorio.

Para este estudio, se han recopilado los datos de rachas máximas de todas las estaciones con registros mensuales desde 1960 hasta 1991, aunque los períodos de observación no son iguales para todas las estaciones. La información corresponde a un total de 97 estaciones extendidas por todo el territorio nacional, en su mayoría ubicadas en aeropuertos, aeródromos y bases aéreas.

\subsection{Tratamiento estadístico de los datos}

Para el tratamiento de toda la información obtenida se ha recurrido a un procedimiento de análisis estadístico de valores extremos conocido como "Distribución de Gumbel". Dicha distribución es una función doble exponencial modificada que se utiliza habitualmente para el estudio de las series de datos extremos que cuantifican fenómenos de la naturaleza, tales como temperaturas, precipitaciones o velocidades de rachas de viento. Su función de distribución es la siguiente:

$$
F(x)=\operatorname{Prob}(w<x)=e^{-e^{-\alpha(x-\mu)}}
$$

siendo $\alpha$ y $\mu$ parámetros definidos como:

$$
\alpha=\frac{S_{n}}{S} \text { y } \mu=\bar{x}-\bar{Y}_{n} \cdot \frac{S}{S_{n}}
$$

donde para valores de la muestra suficientemente grandes:

$$
\begin{gathered}
\bar{Y}_{\mathrm{n}}=\text { Constante de Euler }=0,5772156 \\
\mathrm{~S}_{\mathrm{n}}=\frac{\pi}{6}=1,2825498
\end{gathered}
$$

$\mathrm{S}=$ Desviación típica.

$\overline{\mathrm{x}}=$ Medida aritmética .

Puesto que las mediciones son mensuales, el ajuste a la función de distribución de Gumbel se hace mes a mes, anualizando los resultados obtenidos mediante el teorema de la probabilidad total.

El resultado final proporciona para cada estación meteorológica con información de rachas máximas, las probabilidades de que estas rachas máximas se encuentren en los rangos de velocidad definidos, que van, con saltos de $10 \mathrm{~km} / \mathrm{h}$, descle el intervalo $0-15 \mathrm{~km} / \mathrm{h}$ a $225-235 \mathrm{~km} / \mathrm{h}$.

Adicionalmente, se ha calculado el período de retorno, definido como el intervalo medio de tiempo expresado en años en que el valor extremo alcanza el valor particular $\mathrm{x}$ una vez solamente. Es decir:

$$
\operatorname{Prob}(w \leq x)=1-F(x)=1-e^{-e^{-\alpha(x-\mu)}}
$$

donde, teniendo en cuenta la definición del período de retorno $(\mathrm{T})$, resulta:

$$
T=\frac{1}{1-e^{-e^{-\alpha(x-\mu)}}}
$$

\subsection{Ponderación de los resultados}

Una vez determinada cuál es la probabilidad de que la racha máxima se encuentre en cada uno de los intervalos de velocidad fijados previamente, es preciso establecer un criterio de ponderación lo más objetivo posible, que permita tener en cuenta la capacidad destructiva del viento, que lógicamente será mayor cuanto mayor sea la velocidad del mismo.

El efecto destructivo se ha considerado con la inclusión de la presión dinámica, que equivale a la resis- 
tencia ejercida por un cuerpo a la fuerza del viento y se define como:

$$
\mathrm{W}=\mathrm{v}^{2} / 16
$$

siendo:

$\mathrm{W}=$ Presión dinámica en $\mathrm{kg} / \mathrm{m}^{2}$.

$\mathrm{v}=$ Velocidad del viento en $\mathrm{m} / \mathrm{sg}$.

De la fórmula anterior se deduce que la presión dinámica crece de manera potencial a la velocidad, por lo que duplicar la velocidad $(\mathrm{km} / \mathrm{h})$ produce el efecto de multiplicar por cuatro el valor obtenido para la presión dinámica, triplicar la velocidad multiplicaría por nueve el valor de la presión dinámica, y así sucesivamente.

\subsection{El coeficiente de peligrosidad}

Para asignar un nivel de peligrosidad a cada estación meteorológica, se ha establecido el coeficiente de peligrosidad $\left(\mathrm{C}_{\mathrm{p}}\right)$ que se define como:

$$
C_{p}=\sum P_{i} \cdot w_{i}
$$

siendo:

$C_{p}=$ Coeficiente de peligrosidad

$\mathrm{P}_{\mathrm{i}}=$ Probabilidad de que la racha máxima se encuentre en el intervalo [a,b], siendo a y b valores de velocidad.

$\mathrm{w}_{\mathrm{i}}=$ Valor de la presión dinámica, obtenido de la velocidad correspondiente a la marca de clase del intervalo de velocidad $[a, b]$.

La unidad física del coeficiente de peligrosidad será $\mathrm{kg} / \mathrm{m}^{2}$, por lo que se refiere a un valor de presión. En definitiva, se trata del resultado de la esperanza matemática de la presión dinámica obtenido para cada una de las estaciones meteorológicas analizadas.

\subsection{Definición de los niveles de peligrosidad}

Para fijar los niveles de peligrosidad hay que establecer previamente los umbrales a partir de los cuales se considera que el efecto destructivo del viento puede ser significativo.
Según la escala de vientos de Beaufort, se empiezan a registrar daños materiales en el denominado "DURO" de grado 8, con velocidad del viento entre 62,8 y $74,0 \mathrm{~km} / \mathrm{h}$. Asimismo, Friedman, en diversos estudios, recoge daños en edificaciones por vientos con velocidad superior a $65 \mathrm{~km} / \mathrm{h}$.

En función de lo anterior, se han escogido los 65 $\mathrm{km} / \mathrm{h}$ como velocidad límite a partir de la cual empiezan a producirse daños materiales, por lo que por debajo de este nivel la probabilidad de sufrir daños significativos por efecto del viento, es baja.

De igual manera, y según las mismas escalas, se puede considerar que sobrepasando los $75 \mathrm{~km} / \mathrm{h}$, los daños materiales son ya de consideración. La escala de Beaufort describe los daños sufridos a consecuencia de vientos de más de $75 \mathrm{~km} / \mathrm{h}$ como: "Daños en edificios; caen tejas y chimeneas." Por este motivo, se considera que, a partir de esta velocidad, la probabilidad de sufrir daños por viento es alta. Finalmente, se ha establecido la banda de velocidad comprendida entre los $65 \mathrm{~km} / \mathrm{h}$ y $75 \mathrm{~km} / \mathrm{h}$, como la correspondiente a la hipótesis de peligrosidad media.

Puesto que el análisis de la peligrosidad por estaciones se ha efectuado sobre la presión dinámica, es necesario trasladar los valores de velocidad apuntados a términos de presión, siendo el resultado el que figura en el cuadro propuesto a continuación:

\begin{tabular}{|l|l|l|l|}
\hline $\begin{array}{c}\text { VELOCIDAD } \\
\mathrm{km} / \mathrm{h}\end{array}$ & $\begin{array}{c}\text { VELOCIDAD } \\
\mathrm{m} / \mathrm{sg}\end{array}$ & $\begin{array}{c}\text { COEFICIENTE DE } \\
\text { PELIGROSIDAD } \\
\mathrm{kg} / \mathrm{m}^{2}\end{array}$ & $\begin{array}{c}\text { NIVEL DE } \\
\text { PELIGRO- } \\
\text { SIDAD }\end{array}$ \\
\hline Menos de 65 & Menos de 18,0 & Menos de 20,37 & BAJO \\
\hline 65 a 75 & 18,0 a 20,8 & 20,37 a 27,12 & MEDIO \\
\hline Más de 75 & Más de 20,8 & Más de 27,12 & ALTO \\
\hline
\end{tabular}

\subsection{Asignación de los niveles de peligrosidad por provincias}

Una vez calculados los valores de los coeficientes de peligrosidad por estaciones meteorológicas, es preciso establecer un criterio que permita identificar los resultados obtenidos con provincias, que es la unidad mínima geográfica que se ha escogido.

En este sentido, algunas provipias tienen información de varios observatorios, en tanto que otras tan sólo disponen de una estación con datos de vien- 
tos. En estos casos en los que la falta de información es evidente se ha optado por asignar a toda la provincia el coeficiente de riesgo calculado para el observatorio, ya que las mediciones de éste son, en definitiva, las únicas referencias válidas que sobre el fenómeno de viento se tiene en esa provincia.

En las provincias en las que se dispone de información de más de un observatorio, y en los casos en que no existe homogeneidad en los resultados, se ha asignado el nivel de peligrosidad en función de la tendencia de peligrosidad global observada a la vista de los coeficientes calculados para cada estación meteorológica, prevaleciendo, en último caso, los valores correspondientes a las hipótesis de peligrosidad más pesimistas.

\subsection{Cálculo de los períodos de retorno}

Complementariamente se ha realizado el estudio de los períodos de retorno utilizando también la Distribución de Gumbel. Puesto que el período de retorno debe referirse a una velocidad determinada, se han escogido los $75 \mathrm{~km} / \mathrm{h}$ como velocidad promedio de acuerdo con los límites fijados para el establecimiento de los niveles de peligrosidad.

Asimismo, se incluyen los períodos de retorno correspondientes a $95 \mathrm{~km} / \mathrm{h}$ como velocidad históricamente significativa, según la legislación aseguradora en materia de riesgos extraordinarios.

Del análisis de los valores mínimos y máximos del período de retorno obtenidos para estas velocidades en cada uno de los observatorios estudiados, se puede extraer la siguiente relación con el nivel de peligrosidad asignado:

\begin{tabular}{|l|c|c|}
\hline \multirow{2}{*}{$\begin{array}{c}\text { NIVEL DE } \\
\text { PELIGROSIDAD }\end{array}$} & \multicolumn{2}{c|}{$\begin{array}{c}\text { PERÍODO DE RETORNO } \\
\text { ESTIMADO (años) }\end{array}$} \\
\cline { 2 - 3 } & $75 \mathrm{~km} / \mathrm{h}$ & $95 \mathrm{~km} / \mathrm{h}$ \\
\hline BAJO & Más de 7 & Más de 20 \\
\hline MEDIO & $4-6$ & $8-20$ \\
\hline ALTO & $1-3$ & $1-7$ \\
\hline
\end{tabular}

\section{Conclusiones}

El viento no se puede considerar como un peligro significativo en España, aunque existen cuencas eólicas con vientos predominantes identificados con fines de aprovechamiento energético.

Los vientos predominantes más fuertes soplan en zonas localizadas, como en el Estrecho de Gibraltar, la costa noroeste de Galicia y la costa noreste de Cataluña. Entre las áreas del interior se pueden considerar La Mancha, el valle del Ebro y parte del Sistema Central.

El análisis estadístico aplicado a las series de datos reales ha permitido calcular probabilidades de ocurencia de la racha máxima para cada uno de los intervalos en que se ha estratificado el rango de velocidades, así como conocer el período de retorno para las marcas de clase de cada intervalo. Con carácter general, se puede afirmar que las velocidades de rachas máximas superiores a los 100 $\mathrm{km} / \mathrm{h}$, se han alcanzado alguna vez en prácticamente todos los puntos de la Península Ibérica, variando el período de retorno entre uno y veinte años según el nivel de peligrosidad obtenido tras el ajuste estadístico realizado.

Ha sido preciso asimilar el coeficiente de peligrosidad de una de las estaciones meteorológicas de una provincia o de la tendencia de dicho coeficiente de varias estaciones a toda la provincia. A medida que la red de anemocinemógrafos distribuya un entramado más tupido sobre el territorio nacional será posible diseñar una zonación por peligro de viento más real, a la vez que la introducción de nuevas variables (por ejemplo topografía) será más justificada, dado el futuro nivel de detalle de los datos de base.

\section{NIVEL DE PELIGROSIDAD POR PROVINCIAS}

Provincia

Nivel

peligrosidad

ÁLAVA

ALBACETE

MEDIO

ALTO

MEDIO

ALICANTE

ALMERÍA

MEDIO

ASTURIAS

ALTO

ÁVILA

BAJO

BADAJOZ

MEDIO

BALEARES

MEDIO

BALEARES (MENORCA)

ALTO

BARCELONA

BURGOS

MEDIO

ALTO

CÁCERES

CÁDIZ

MEDIO

ALTO

MEDIO

CASTELLÓN

ALTO

CEUTA

BAJO 


\section{HUELVA}

HUESCA

JAÉN

LEÓN

LÉRIDA

mapa de peligrosidad por vientos fuertes

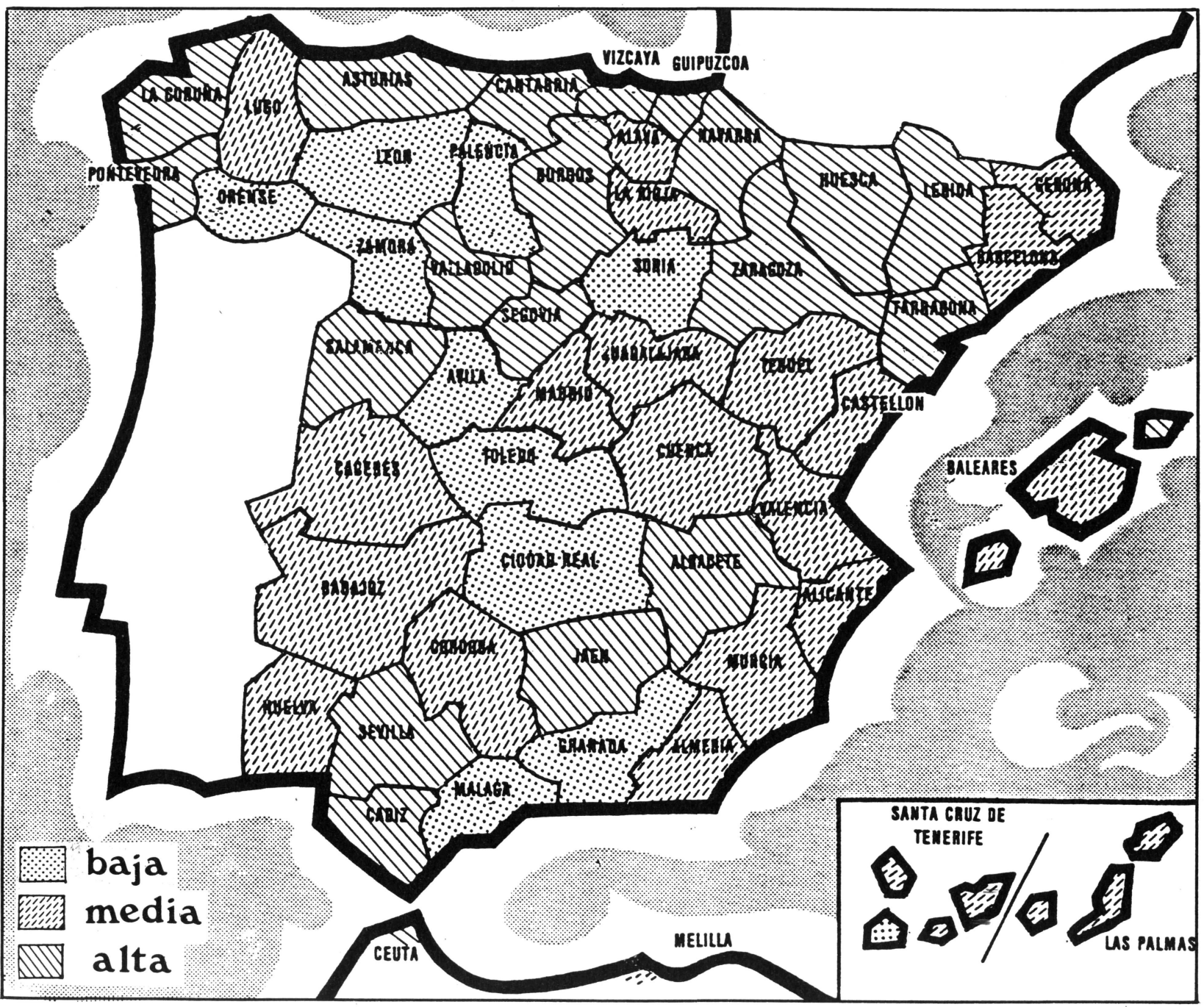




\section{RELACIÓN DE OBSERVATORIOS}

\begin{tabular}{|c|c|}
\hline Provincia & Estación climatológica \\
\hline ÁLAVA & A. VITORIA \\
\hline ALBACETE & $\begin{array}{l}\text { B. A. LOS LLANOS } \\
\text { OBSERVATORIO }\end{array}$ \\
\hline ALICANTE & $\begin{array}{l}\text { A. EL ALTET } \\
\text { CIUDAD JARDÍN }\end{array}$ \\
\hline ALMERÍA & $\begin{array}{l}\text { AEROPUERTO } \\
\text { CIUDAD JARDÍN }\end{array}$ \\
\hline ASTURIAS & $\begin{array}{l}\text { A. OVIEDO (EL CRISTO) } \\
\text { GIJÓN } \\
\text { A. AVILES }\end{array}$ \\
\hline ÁVILA & OBSERVATORIO \\
\hline BADAJOZ & B. A. TALAVERA LA REAL \\
\hline BALEARES & $\begin{array}{l}\text { PALMA DE MALLORCA } \\
\text { POLLENSA } \\
\text { A. SON SAN JUAN } \\
\text { MAHÓN (MENORCA) } \\
\text { A. ES CODOLA (IBIZA) }\end{array}$ \\
\hline BARCELONA & $\begin{array}{l}\text { CENTRO METEOROLÓGICO } \\
\text { ROURA } \\
\text { A. PRAT DE LLOBREGAT } \\
\text { MONTSENY } \\
\text { GRANOLLERS }\end{array}$ \\
\hline BURGOS & B. A. VILLAFRÍA \\
\hline CÁCERES & OBSERVATORIO \\
\hline CÁDIZ & $\begin{array}{l}\text { SAN FERNANDO } \\
\text { TARIFA } \\
\text { PUERTO TIERRA }\end{array}$ \\
\hline CASTELLÓN & OBSERVATORIO \\
\hline CEUTA & OBSERVATORIO \\
\hline CIUDAD REAL & OBSERVATORIO \\
\hline CÓRDOBA & OBSERVATORIO \\
\hline CORUÑA, LA & $\begin{array}{l}\text { OBSERVATORIO } \\
\text { A. LABACOLLA } \\
\text { A. ALVEDRO } \\
\text { MONTEVENTOSO }\end{array}$ \\
\hline CUENCA & OBSERVATORIO \\
\hline GERONA & AEROPUERTO \\
\hline GRANADA & $\begin{array}{l}\text { LA CARTUJA } \\
\text { B. A. ARMILLA } \\
\text { AEROPUERTO }\end{array}$ \\
\hline GRAN CANARIA & $\begin{array}{l}\text { LA LUZ } \\
\text { A. GANDO (GRAN CANARIA) } \\
\text { A. ARRECIFE (LANZAROTE) }\end{array}$ \\
\hline GUADALAJARA & $\begin{array}{l}\text { OBSERVATORIO } \\
\text { MOLINA DE ARAGÓN }\end{array}$ \\
\hline GUIPÚZCOA & $\begin{array}{l}\text { A. FUENTERRABÍA } \\
\text { IGUELDO }\end{array}$ \\
\hline HUELVA & RONDA ESTE \\
\hline HUESCA & MONTFLORITE \\
\hline
\end{tabular}

$\begin{array}{ll}\text { Provincial } & \text { Estación climatológica } \\ \text { JAÉN } & \text { INSTITUTO } \\ \text { LEÓN } & \text { B. A. VIRGEN DEL CAM } \\ \text { LÉRIDA } & \text { OBSERVATORIO } \\ \text { LOGRONOO } & \text { B. A. AGONCILLO } \\ \text { ESTACIÓN AGROMETEO } & \text { PUNTO CENTRO } \\ \text { LUGO } & \text { A. BARAJAS } \\ \text { MADRID } & \text { B. A. CUATRO VIENTO } \\ & \text { B. A. GETAFE } \\ & \text { RETIRO } \\ & \text { PUERTO DE NAVACER } \\ & \text { CIUDAD UNIVERSITAR } \\ & \text { B. A. TORREJÓN } \\ \text { MÁLAGA } & \text { A. EL ROMPEDIZO } \\ \text { MELILLA } & \text { AEROPUERTO } \\ \text { MURCIA } & \text { B. A. ALCANTARILLA } \\ & \text { B. A. SAN JAVIER } \\ & \text { GUADALUPE }\end{array}$

NAVARRA

A. NOAIN

ORENSE

OBSERVATORIO

PALENCIA

OBSERVATORIO

PONTEVEDRA

MARÍN

PEINADOR

SALAMANCA IOATO

B. A. MATACÁN

MUÑOVELA

SANTANDER

OBSERVATORIO

A. PARAYAS

SEGOVIA

OBSERVATORIO

SEVILLA

SORIA

A. SAN PABLO

OBSERVATORIO CEDER-LUBIA

TARRAGONA REUS

VANDELLÓS

TENERIFE

A. EL HIERRO

A. LOS RODEOS

SANTA CRUZ

A. REINA SOFÍA

IZAǸA

A. MAZO (LA PALMA)

TERUEL

OBSERVATORIO

TOLEDO

OBSERVATORIO

A. MANISES

VALLADOLID

UNIVERSIDAD

B. A. VILLANUBLA

VIZCAYA

A. SONDICA

ZAMORA

ZARAGOZA
OBSERVATORIO

A. SANJURJO 


\section{BIBLIOGRAFÍA}

- Ascaso Liria, A., y Casals Marcen, M: "Vocabulario de Términos Meteorológicos y Ciencias Afines”, Ed. Instituto Nacional de Meteorología (INM), Madrid, 1986.

- Blanco García, A.: "Distribución de valores extremos (Períodos de Retorno)", Servicio de Climatología del INM, mayo, 1983.

— Font Tullot, I.: "Climatología de España y Portugal”, Madrid, $1983 \mathrm{~b}$.

- Friedman, D. G.: "Natural hazard risk Assessment for an insurance program." en "The Geneva Papers on Risk and Insurance”, volumen 9, número 30, Ginebra, enero, 1984.

- García de Pedraza, L.: "Comunicación personal."

- Gumbel, E.: "Statistical Theory of Extreme Values and some practical applications." United States Department of Commerce. National Bureau of Standards, febrero 1954.

- Instituto Nacional de Meteorología: "Calendario Meteorofenológico.” Años 1951 y 1958. Servicio Meteorológico Nacional. Madrid.

- Instituto Nacional de Meteorología: "Mapa Eólico Nacional. Análisis del viento para aprovechamiento energético." Ed. INM Madrid, 1988.

- Ministerio de Obras Públicas y Urbanismo (MOPU): "Norma Básica de la Edificación” NBE-AE-88. "Norma Tecnológica de la Edificación” NTE-ECV-88. "Cargas de viento", Madrid, 1988.
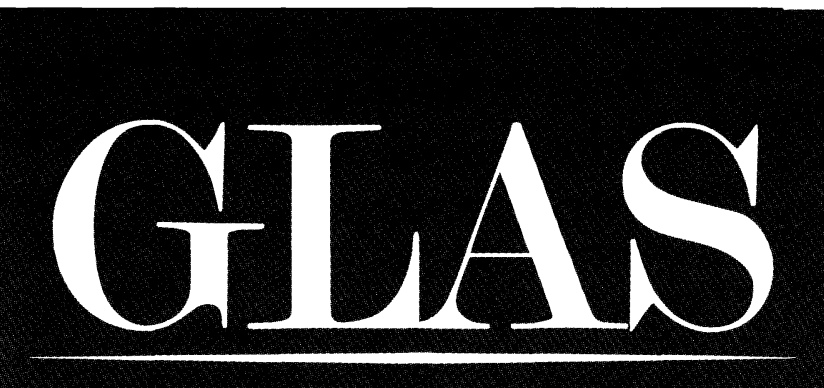

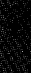

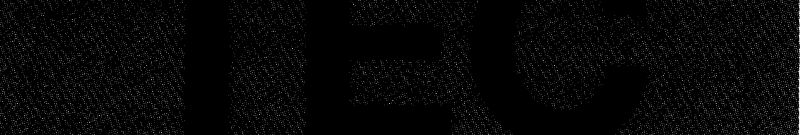

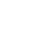

